\title{
BMJ Open Psychiatric disorders among people with cancer in low- and lower-middle- income countries: study protocol for a systematic review and meta-analysis
}

\author{
Zoe J Walker, ${ }^{1,2}$ Michael P Jones, ${ }^{3,4}$ Arun V Ravindran ${ }^{1,2}$
}

To cite: Walker ZJ, Jones MP, Ravindran AV. Psychiatric disorders among people with cancer in low- and lowermiddle-income countries: study protocol for a systematic review and meta-analysis. BMJ Open 2017;7:e017043. doi:10.1136/ bmjopen-2017-017043

- Prepublication history and additional material for this paper are available online. To view these files please visit the journal online (http://dx.doi. org/10.1136/bmjopen-2017017043).

Received 28 March 2017 Revised 16 June 2017 Accepted 3 July 2017

\section{(a) CrossMark}

${ }^{1}$ Department of Psychiatry, University of Toronto, Toronto, Ontario, Canada

${ }^{2}$ Centre for Addiction and Mental Health, Toronto, Ontario, Canada ${ }^{3}$ Department of Radiation Oncology, University of Toronto, Toronto, Ontario, Canada ${ }^{4}$ Princess Margaret Cancer Centre, Toronto, Ontario, Canada

Correspondence to

Arun V Ravindran;

Arun.Ravindran@camh.ca

\section{ABSTRACT}

Introduction Cancer is a rapidly growing public health problem in low- and lower-middle-income countries (LLMICs). There is evidence from upper-income countries that comorbid mental illness is common and can adversely impact cancer outcomes. Little is known about this burden in LLMICs. This systematic review has two aims. The first is to review the prevalence and patterns of psychiatric comorbidity in adults with cancer in LLMICs. The second is to review psychiatric treatment outcomes in this population. Methods and analysis The review will be reported according to the Preferred Reporting Items for Systematic Review and Meta-Analysis (PRISMA) guidelines. A systematic search of electronic databases (MEDLINE, Psyclnfo, Embase and CINAHL) will be conducted. Studies will be included if they report the prevalence of psychiatric comorbidity, or if they evaluate psychiatric treatment outcomes, in adults with cancer living in LLMICs. The search will be limited to studies published in peer-reviewed journals between March 2002 and March 2017. The reference lists of included studies will be hand searched. Critical appraisal will be performed using Quality Assessment Tools from the National Institute of Health. Pooled prevalence meta-analysis is planned.

Ethics and dissemination Ethics approval is not required as no primary data will be collected. The results will be presented at conferences and published in a peerreviewed journal.

Systematic review registration PROSPERO CRD42017057103.

\section{BACKGROUND}

The World Health Organization (WHO) estimates that new cancer cases will increase from 14.1 million in 2012 to 21.7 million in $2030 .^{1}$ The greatest increase will occur in low- and lower-middle-income countries (LLMICs), ${ }^{2}$ where non-communicable diseases have already replaced communicable diseases as the greatest cause of morbidity and mortality. ${ }^{3}$ This growth is driven by numerous factors: population growth, increasing life expectancy, greater detection and reporting, lifestyle risk factors, and infection by oncogenic viruses,

\section{Strengths and limitations of this study}

- The protocol follows the established Preferred Reporting Items for Systematic Reviews and MetaAnalysis Protocols (PRISMA-P) reporting guidelines. Similarly, PRISMA guidelines will be used in the preparation of the systematic review.

- The review will involve a comprehensive search strategy and a robust screening process.

- The quality of included articles will be independently assessed by two authors.

- Reporting bias may occur due to the exclusion of articles published in languages other than English, articles published prior to 2002 and unpublished articles.

- The number of reports found may be limited, and high heterogeneity may prevent a meta-analysis.

such as hepatitis B and C, human papillomavirus or Helicobacter pylori. ${ }^{4-6}$

Emotional distress commonly occurs during the cancer trajectory and ranges from subclinical distress to mental illness. It is well documented that psychiatric comorbidity is associated with reduced quality of life, ${ }^{7}$ as well as poorer cancer outcomes including shorter survival. ${ }^{8}$ Hence, the International Federation of Psycho-oncology Societies endorses distress as the 'sixth vital sign' in cancer ${ }^{9}$ and advocates for psychosocial support to be integrated into cancer care. ${ }^{10}$

The majority of studies evaluating the prevalence of psychiatric comorbidity in cancer have been conducted in upper-income countries. A study of 2141 participants in Germany found a 12-month prevalence of mental disorders of $39.4 \% .^{11}$ Meta-analyses have estimated the pooled prevalence of comorbid mental disorders as being $32 \%,{ }^{12}$ comorbid mood disorders to range from $30 \%$ to $40 \%^{13}$ and comorbid depression to range from $8 \%$ to $24 \% .{ }^{14}$ In China, an upper-middle-income country, albeit with significant intra-country variation, a 
meta-analysis found the prevalence of depression and anxiety to be $54.9 \%$ and $49.7 \%$, respectively. ${ }^{15}$ Diagnosis of mental disorders was made using clinical interviews $^{12-15}$ or self-report instruments. ${ }^{1415}$

Patients with cancer in LLMICs, compared with upper-income countries, often face significant additional challenges: cultural barriers to care may exist, diagnosis is often late and treatment options can be limited and costly. ${ }^{16}$ There is often poor access to pain medication and poorer survival rates. ${ }^{4}{ }^{17}$ In upper-income countries, socioeconomic factors such as low income ${ }^{18}$ and low education ${ }^{18} 19$ have been found to be associated with increased depressive symptoms among patients with cancer. It is unclear how the challenges faced by those in LLMICs impact on psychological symptoms.

Mental health interventions in people with cancer result in improved mental health and quality of life outcomes ${ }^{2021}$ and reduction in medical costs to the healthcare system. ${ }^{21}$ Generalisability to LLMICs may be limited due to cultural, socioeconomic and health system differences. In LLMICs, cancer care itself is under-resourced and psycho-oncology services are often scarce or non-existent. ${ }^{10}$ It is important for interventions to be evidence based to justify the allocation of limited resources.

Improved understanding of psychiatric comorbidity in people with cancer in LLMICs will raise awareness of the burden of disease and may inform the development of national cancer care plans. In countries where there is no national mental health programme, some mental healthcare needs may be addressed by embedding psychological care into medical care. ${ }^{22}$

The aims of this systematic review are to describe, among adults with cancer living in LLMICS:

- the prevalence and determinants of psychiatric comorbidity

- the efficacy of mental health interventions.

\section{METHODS}

The research protocol follows the Preferred Reporting Items for Systematic Reviews and Meta-Analysis Protocols (PRISMA-P) guidelines. ${ }^{23}$

\section{Eligibility criteria}

Studies will be selected according to the following criteria:

- All study designs will be included, except for case reports or small case series under 20 patients.

- Study participants must be:

- adulthumans (18 years or older). Studies evaluating both adults and children will be included if the data for adults is presented separately.

- living in LLMICs, as defined by the World Bank country income categories. Studies evaluating countries from different income categories will be included if the data for LLMICs is presented separately.

- people with a diagnosis of cancer.

- The study must report either the prevalence of psychiatric comorbidity or assess the outcomes of psychi- atric interventions. Psychiatric comorbidity must be assessed by either a validated instrument or clinical interview. Validation studies will be excluded.

- The search will be limited to the English language and to peer-reviewed articles.

- The date range will be limited from March 2002 to March 2017.

\section{Information sources and search strategy}

We will search MEDLINE, PsycInfo, Embase and CINAHL. We will develop a literature search strategy using a combination of medical subject headings and keywords. In order to remain relevant to current practice, we intend to calculate a contemporary pooled prevalence and focus on up-to-date interventions. We will limit the search to studies published after March 2002. This reflects the time period when non-communicable disease became a health priority for LLMICs in response to the epidemiological shift from communicable to non-communicable diseases. ${ }^{24}{ }^{25}$ Additionally, it allows for the wide adoption of the Diagnostic and Statistical Manual IV and the International Statistical Classification of Diseases, Tenth Revision diagnostic systems in order to limit phenotypic heterogeneity. ${ }^{26}$

The specific search strategy will be created in consultation with an experienced Health Sciences librarian. The draft MEDLINE search strategy is included in online appendix 1 . It will be adapted to the syntax and subject headings of the other databases. The reference lists of included studies will also be screened against the eligibility criteria.

\section{Data management and selection process}

Literature search results will be uploaded to Endnote $\mathrm{X} 8$, a software program for managing references and citations. Two authors will independently screen the titles and abstracts against the inclusion criteria and create a preliminary list of articles. One author will retrieve and screen the full text of these articles. We will record the reasons for exclusion. The authors will not be blind to the authors or journal titles. The process will be documented in the PRISMA flow diagram. Disagreements will be resolved through consensus or by adjudication by a third author.

\section{Data collection process}

Using standardised forms, one author will extract the data from the included studies. The data will be crosschecked by a second author. The key outcome is the prevalence of depression. Secondary outcomes will be the prevalence of anxiety and other mental disorders. Extracted information will include: author, year of publication, country, study design, percentage women, type of cancer, time since diagnosis, measurement tools and outcomes, intervention if relevant, and determinants of psychiatric comorbidity. For treatment studies, details regarding the intervention, control group and effect of the intervention will also be extracted. We will 
contact corresponding authors for clarification or for missing data.

\section{Quality assessment}

Quality assessment for each included study will be independently undertaken by two authors using the National Institute of Health's National Heart Lung Brain Institute Quality Assessment Tools. ${ }^{27}$ The tool provides guidance for evaluating the internal validity of the study and for translating this to risk of bias. Each study will be rated as being at low, medium or high risk of bias. Disagreements will be resolved by consensus or by adjudication by a third author. We will assess publication bias using a funnel plot.

\section{Data synthesis}

Data on the prevalence and determinants of psychiatric comorbidity and data on interventions will be synthesised in separate tables. A systematic narrative synthesis will be presented for all tables.

Meta-analysis of prevalence data will be undertaken. Study heterogeneity will be calculated using I2statistic. Provided that depression has been evaluated in at least two studies, the overall pooled prevalence will be calculated using a random effects meta-analysis. Similarly, if other individual mental disorders have been evaluated in at least two studies, the overall pooled prevalence of these will also be calculated using a random effects meta-analysis. Subgroup analyses may be made based on geographical location, method of diagnosis and risk of bias.

The study will be reported according to the PRISMA guidelines. $^{28}$

Acknowledgements We would like to extend our thanks to Sharon Bailey, medical librarian at the Centre for Addiction and Mental Health, for her assistance with developing our search strategy.

Contributors ZJW and AVR conceived the study. ZJW registered with PROSPERO. MPJ assisted in protocol design. ZJW drafted the protocol. AVR and MPJ reviewed and edited the protocol. All authors reviewed and approved the final manuscript. ZJW is the guarantor.

Funding ZJW's Fellowship at the University of Toronto has been funded by a New South Wales Institute of Psychiatry (NSWIOP) Special Training Fellowship grant. The NSWIOP is not involved in any other aspect of the project.

Competing interests None declared.

Provenance and peer review Not commissioned; externally peer reviewed.

Open Access This is an Open Access article distributed in accordance with the Creative Commons Attribution Non Commercial (CC BY-NC 4.0) license, which permits others to distribute, remix, adapt, build upon this work non-commercially, and license their derivative works on different terms, provided the original work is properly cited and the use is non-commercial. See: http://creativecommons.org/ licenses/by-nc/4.0/

(C) Article author(s) (or their employer(s) unless otherwise stated in the text of the article) 2017. All rights reserved. No commercial use is permitted unless otherwise expressly granted.

\section{REFERENCES}

1. Ferlay J, Soerjomataram I, Ervik M, et al. GLOBOCAN 2012 v1.0, cancer incidence and mortality worldwide: IARC CancerBase No. 11
(Internet). International Agency for Research on Cancer: Lyon, 2013. http://globocan.iarc.fr/ (accessed 23 Mar 2017).

2. World Health Organization. Global status report on noncommunicable diseases 2010: description of the global burden of NCDs, their risk factors and determinants. Geneva: World Health Organization, 2011.

3. Global burden of disease study 2015. global burden of disease study 2015 (GBD 2015) Life Expectancy, All-Cause and CauseSpecific Mortality 1980-2015. Seattle: Institute for Health Metrics and Evaluation 2016.

4. Sloan FA, Gelband H, eds. Cancer control opportunities in low- and middle-income countries (Internet). Washington (DC): National Academies Press (US), 2007. https://www.ncbi.nlm.nih.gov/books/ NBK54030/(accessed 23 Mar 2017).

5. Kanavos P. The rising burden of Cancer in the developing world. Ann Oncol 2006;17 Suppl 8:viii15-viii23.

6. Cancer Atlas. Infection (Internet). Georgia: American Cancer Society, 2014. (accessed 12 May 2017).

7. Skarstein J, Aass N, Fosså SD, et al. Anxiety and depression in Cancer patients: relation between the Hospital anxiety and depression Scale and the European Organization for Research and treatment of Cancer core Quality of Life Questionnaire. J Psychosom Res 2000;49:27-34.

8. Pinquart M, Duberstein PR. Depression and Cancer mortality: a meta-analysis. Psychol Med 2010;40:1797-810.

9. Bultz BD, Travado L, Jacobsen PB, et al. 2014 President's plenary international psycho-oncology society: moving toward cancer care for the whole patient. Psychooncology 2015;24:1587-93.

10. Grassi L, Watson M, IFoP-OS co-authors; IPOS Federation of Psycho-Oncology Societies' co-authors. Psychosocial care in Cancer: an overview of psychosocial programmes and national Cancer plans of countries within the International Federation of Psycho-Oncology Societies. Psychooncology 2012;21:1027-33.

11. Kuhnt S, Brähler E, Faller $\mathrm{H}$, et al. Twelve-Month and Lifetime Prevalence of Mental Disorders in Cancer Patients. 1423-0348 (Electronic)

12. Singer S, Das-Munshi J, Brähler E. Prevalence of mental health conditions in Cancer patients in acute care--a meta-analysis. Ann Oncol 2010;21:925-30.

13. Mitchell AJ, Chan M, Bhatti $\mathrm{H}$, et al. Prevalence of depression, anxiety, and adjustment disorder in oncological, haematological, and palliative-care settings: a meta-analysis of 94 interview-based studies. Lancet Oncol 2011;12:160-74.

14. Krebber AM, Buffart LM, Kleijn G, et al. Prevalence of depression in Cancer patients: a meta-analysis of diagnostic interviews and selfreport instruments. Psychooncology 2014;23:121-30.

15. Yang YL, Liu L, Wang Y, et al. The prevalence of depression and anxiety among chinese adults with Cancer: a systematic review and meta-analysis. BMC Cancer 2013;13:393.

16. Cazap E, Magrath I, Kingham TP, et al. Structural barriers to diagnosis and treatment of Cancer in low- and MiddleIncome Countries: the urgent need for scaling up. $J$ Clin Oncol 2016;34:14-19.

17. Horton S, Gauvreau CL. Cancer in low- and middle-income countries: an economic overview. In: Gelband H, Jha P, Sankaranarayanan R, Cancer: disease control priorities, third edition (Volume 3) (Internet). Washington(DC): The International Bank for Reconstruction and Development / The World Bank, 2015. https://www.ncbi.nlm.nih.gov/books/NBK343620/(accessed 23 Mar 2017).

18. Zainal NZ, Nik-Jaafar NR, Baharudin A, et al. Prevalence of depression in breast Cancer survivors: a systematic review of observational studies. Asian Pac J Cancer Prev 2013;14:2649-56.

19. Sehlen S, Lenk M, Herschbach P, et al. Depressive symptoms during and after radiotherapy for head and neck Cancer. Head Neck 2003:25:1004-18.

20. Osborn RL, Demoncada AC, Feuerstein M. Psychosocial interventions for depression, anxiety, and quality of life in Cancer survivors: meta-analyses. Int J Psychiatry Med 2006;36:13-34.

21. Carlson LE, Bultz BD. Benefits of psychosocial oncology care: improved quality of life and medical cost offset. Health Qual Life Outcomes 2003;1:8.

22. Patel V. Belkin gs fau - Chockalingam A, Chockalingam A Fau Cooper J, Cooper J Fau - Saxena S, Saxena S fau - Unutzer J, Unutzer J. Grand challenges: integrating mental health services into priority health care platforms. 1549-1676.

23. Moher D, Shamseer L, Clarke M, et al. PRISMA-P Group. Preferred reporting items for systematic review and meta-analysis protocols (PRISMA-P) 2015 statement. Syst Rev 2015;4:1.

24. Organization WH. Preventing chronic diseases: a vital investment. Geneva: World Health Organization, 2005:200. 
25. Yach D, Hawkes C. Towards a WHO long - term strategy for prevention and control of leading chronic diseases. Geneva: World Health Organization, 2004:86.

26. Andrews G, Slade T, Peters L. Classification in psychiatry: ICD-10 versus DSM-IV. Br J Psychiatry 1999;174:3-5. 0007-1250.

27. Heart N. Lung and Blood Institute. Study quality assessment tools [Internet]. Bethesda: national Heart, lung, and Blood Institute,
2014. https://www.nhlbi.nih.gov/health-pro/guidelines/in-develop/ cardiovascular-risk-reduction/tools (accessed 23 Mar 2017).

28. Moher D, Liberati A, Tetzlaff J, et al. Preferred reporting items for systematic reviews and meta-analyses: the PRISMA Statement. BMJ 2009:339. 